Unravelling the projective thinking: a look from the creative phenomenon at three components of architectural design

\section{| RESUMEN |}

Usando técnicas de estudios de protocolo, esta investigación observó cómo en la mente de 16 arquitectos se desarrollan tres procesos cognitivos: la imaginería visual, la percepción visual y la activación de conocimiento semántico alojado en la memoria de largo plazo, que interactuaban durante la creación de propuestas de diseño arquitectónico. Los resultados sugieren que el alto rendimiento en términos del nivel de creatividad que mostraron los proyectos mejor evaluados obedece al uso intensivo de conocimiento semántico de sus autores y no a sus habilidades de procesamiento visual -perceptual o imaginado- como podría pensarse.

\section{| ABSTRACT}

Using techniques of studies of protocol, this research observed three cognitive processes developed in the minds of 16 architects, which are: visual imagery, visual perception, and activation of semantic knowledge, all housed in the long-term memory that interacted during the creation of architectural design proposals. The results suggest that the high performance in terms of the level of creativity, shown by the best rated projects, correspond to the authors' intensive use of semantic knowledge, rather than to their visual processing skills - perceptual or imagined-as one might think.

PEDRO SOZA R.*

\title{
Desenredando el pensamiento proyectual: una mirada desde el fenómeno creativo a tres componentes del diseño arquitectónico
}

\section{INTRODUCCIÓN}

Esta investigación revisa tres de las operaciones mentales que permiten que los proyectos de diseño nazcan de la mente de un diseñador: la percepción visual, la imaginación visual, y la activación de conocimiento guardado en la memoria de largo plazo. La selección de estas operaciones se basó en dos criterios. Primero, los tres procesos son clave para la cognición humana, lo que ha sido demostrado y consolidado durante más de 60 años de investigación científica en campos como la psicología cognitiva, neurociencias, y las ciencias cognitivas. Segundo, si bien a estas alturas estos procesos mentales están claramente definidos, el rol específico que cada uno de ellos cumple durante el aprendizaje y la práctica profesional del diseño, en este caso arquitectónico, está lejos de ser comprendido (Eastman, 2001).

\section{ANTECEDENTES PREVIOS: COGNICIÓN HUMANA EN LA PRÁCTICA DEL DISEÑO}

\section{PERCEPCIÓN VISUAL, IMAGINERÍA MENTAL Y ESPACIO}

Es común pensar que arquitectos y diseñadores, en general, poseen habilidades espaciales y visuales que se encuentran altamente desarrolladas. De hecho, es habitual también escuchar este tipo de predicamentos en facultades y escuelas de arquitectura y diseño, y hasta creer que la educación en diseño desarrolla dichas habilidades cognitivas. Sin embargo, al revisar los antecedentes

\footnotetext{
Arquitecto de la Universidad de Chile en 1997. Desde el año 1997 al año 2001 trabajó en la oficina de Mario Paredes y Arquitectos Asociados. El año 2004, regresó como académico invitado a la Universidad de Chile y se unió a SIGraDi, la Sociedad Iberoamericana de Gráfica Digital. El año 2005, fue invitado como miembro al jurado de la Bienal Internacional de Arquitectura de Miami Beach, y al comité científico de la SIGraDi en el que ha permanecido hasta la fecha. El año 2006 presidió la X Conferencia Internacional de SIGraDi, realizada en la Facultad de Arquitectura y Urbanismo de la Universidad de Chile. Del 2007 al 2009, como presidente electo de la Sociedad Iberoamericana de Gráfica Digital, fue invitado a formar parte del jurado internacional para FEIDAD, el Premio Internacional de Diseño Arquitectónico del Lejano Oriente, en Taiwán. Galardonado con una beca Fulbright, el año 2009 ingresó al programa de doctorado en cognición del diseño y diseño computacional en el Instituto Tecnológico de Georgia, Georgia Tech. Actualmente, la investigación del profesor Soza se centra en procesos cognitivos en diseño, específicamente en entender el papel que las representaciones mentales -procedurales y semánticas- de conocimiento en diseño juegan tanto en la formulación de problemas de diseño como en la toma de decisiones proyectuales.
} 


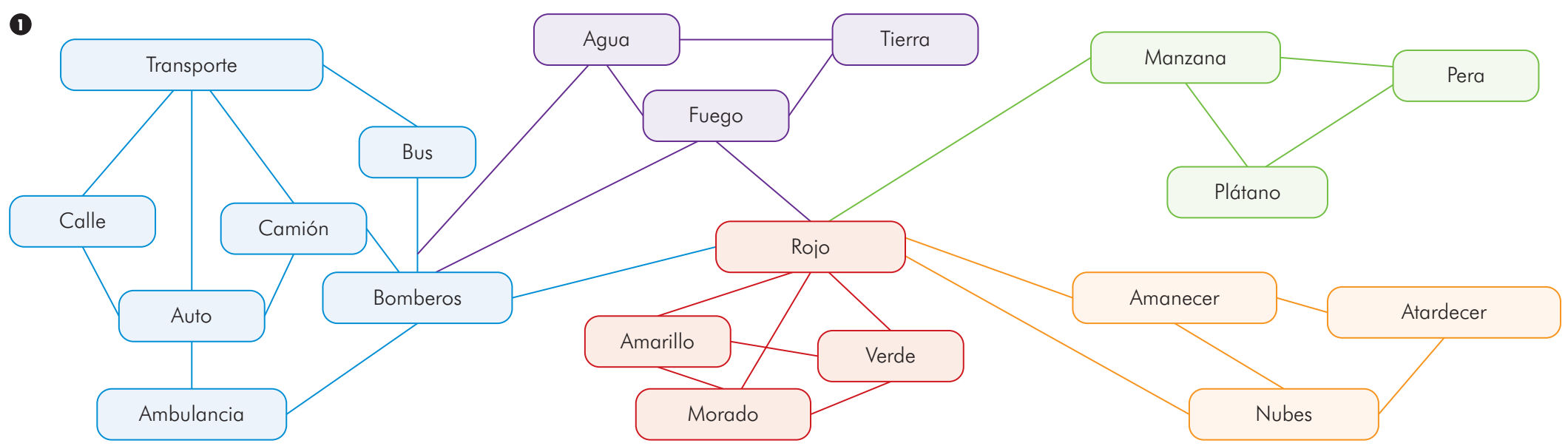

que existen sobre «habilidades espaciales y visuales»-independientemente de lo que arquitectos y diseñadores entendamos por ellas- nos encontramos con que distintos circuitos neuronales alojados en distintas zonas del cerebro humano representan y procesan información espacial por un lado e información visual por el otro (Finke, 1989; Kosslyn, 1994). La importancia de esta separación sugiere que a nivel cognitivo la representación y el procesamiento de información visual y espacial son de naturalezas distintas (Farah, 1994).

Así, mientras el procesamiento visual detecta y representa características como color, forma y movimiento, el procesamiento espacial detecta y representa distancias, dimensiones y orientación. Si bien esta separación funcional abre la discusión filosófica-ajenas a este estudiosobre la naturaleza del espacio según lo entienden los arquitectos, es interesante señalar que existe un tercer tipo de representación mental relacionado con la cognición visual que comúnmente no tomamos en cuenta, las representaciones motoras (representaciones de acción o movimiento que se alojan y procesan en la corteza motora del cerebro y que envían señales eléctricas a los músculos a fin de producir movimiento). Años de investigación en cognición y neurociencias han demostrado que la naturaleza del procesamiento visual ejecutado por artistas y por diseñadores está vinculada a las habilidades de ejecución motora de dichos artistas y diseñadores (Kozbelt, 2001; Seeley and
Kozbelt, 2008; Pignocchi, 2010). Dando por sentado las contribuciones de Kozbelt, Seeley y Kozbelt y Pignocchi, este estudio propuso revisar el rol que juegan las «habilidades visuales», concretamente la percepción visual y la imaginación espacial, en la práctica del diseño arquitectónico.

\section{REPRESENTACIONES SEMÁNTICAS: EL STOCK DE CONOCIMIENTO DEL DISEÑO}

Un segundo tipo de predicamentos comúnmente escuchados en el discurso arquitectónico son los relacionados a los referentes conceptuales. Es importante esclarecer que, técnicamente, lo que los arquitectos y diseñadores entendemos por referentes conceptuales corresponde a representaciones mentales alojadas en la memoria de largo plazo y catalogadas como «semánticas» en la literatura especializada. Esto, no es nuevo. Desde mediados del siglo $x X$, gracias a los experimentos llevados a cabo por Tulving en el campo de la psicología cognitiva, es sabido que la memoria de largo plazo almacena por un lado representaciones semánticas -relacionadas a qué son las cosas- y por otro representaciones procedurales -relacionadas a cómo hacemos las cosas- (Tulving, 1972), entre otros tipos de representaciones como son las episódicas, las perceptuales, las de condicionamiento, y las no asociativas (Gazzaniga, lvry y Mangum,
1998). De la misma forma, es sabido cómo construimos y consolidamos las representaciones de contenido semántico en la memoria de largo plazo (teoría de reconocimiento de los objetos, Warrington, 1978), y cómo se activan y llamamos dichas representaciones (teoría de procesamiento semántico, Quillan, 1968) (Figura 1) cuando las necesitamos, así como las reglas que gobiernan el proceso de activación de recuerdos (Collins y Loftus, 1975). En conjunto, estos estudios ofrecen una explicación hipotética al porqué diseñadores y arquitectos trabajamos con asociaciones mentales catalogadas como obvias, por ejemplo «entrada y hall están juntos» al mismo tiempo que experimentemos asociaciones mentales lejanas o novedosas, del tipo «el proyecto va a comportarse como un árbol». Particularmente las teorías de Quillan y Collins y Loftus sugieren que el fenómeno creativo en la práctica del diseño surge producto de la activación entre representaciones mentales semánticas -que pueden ser distintas en naturaleza: visuales, sonoras, motoras- especialmente lejanas en la red que conforma el stock de conocimiento semántico que arquitectos o diseñadores poseemos. Dentro de este marco teórico, la selección de representaciones semánticas como variables en estudio se fundamentó en comprender el rol específico que dichas representaciones, en cuanto referentes conceptuales, tienen para la proposición de soluciones en proyectos de diseño. 


\section{CREATIVIDAD EN DISEÑO}

Una de las condiciones no escritas en las disciplinas del diseño es que sus resultados son originales e irrepetibles. La noción de originalidad está estrechamente vinculada a la de creatividad. Investigadores en el campo de cognición del diseño sostienen que la creatividad es un fenómeno vinculado a los procesos cognitivos que dan origen a los objetos de diseño (Dorst \& Cross, 2001; Roseman \& Gero, 1993). Particularmente Roseman y Gero han sugerido que el fenómeno creativo emerge como resultado de cinco procedimientos mentales: combinación, transformación o mutación, analogía, primer principio y descubrimiento. Mientras que los cuatro primeros operan sobre aspectos existentes del diseño, el último surge como una nueva propiedad o particularidad en los procesos de diseño (Roseman \& Gero, 1993). Esta idea propuesta por Roseman y Gero puede ser explicada por la teoría de activación semántica propuesta por Quillan y desarrollada por sus discípulos Collins y Loftus. Es más, dichas ideas nos permiten proponer una explicación a cómo es que los arquitectos y diseñadores asignan propiedades semánticas a los espacios y objetos que diseñan, lo que a su vez está alineado con los hallazgos de Kozbelt sobre la naturaleza del procesamiento visual y sus posibles correlatos semánticos en artistas y diseñadores. Adicionalmente es importante destacar que en conjunto estas teorías y hallazgos son congruentes con los resultados de los estudios sobre expertiz y desarrollo de profesionales expertos liderados por Ericsson en los noventa, los que establecieron que expertos en cualquier campo llegan a ser tales después de al menos 10 años de práctica consecutiva (Ericsson, Krampe \& Tesch-Roemer, 1993; Ericsson \& Charness, 1994). Particularmente de interés para este estudio es la sugerencia de que el rendimiento extraordinario de expertos, arquitectos para los efectos de este estudio, y el surgimiento de la creatividad en la práctica de sus distintos oficios descansarían en la vasta red de conocimiento semántico que dichos expertos desarrollan durante 10 años o más de práctica profesional (Ericsson \& Charness, 1994), lo que también ha sido recogido en el ámbito de diseño por distintos autores (Cross, 1997; Dorst \& Cross, 2001 ; Lawson 2001, $2005,2009)$ pero no comprobado -al menos en conocimiento de este autor- con estudios empíricos a la fecha.

\section{HIPÓTESIS Y PREGUNTAS DE INVESTIGACIÓN}

En base al problema de investigación y el marco teórico propuesto, el estudio formuló las siguientes preguntas de investigación e hipótesis correspondientes:

1. ¿̇Tienen los arquitectos un rendimiento cognitivo superior al resto de la población en percepción visual, imaginería visual y formulación conceptual?

H1. Los arquitectos tienen un rendimiento cognitivo superior al común de la población en test de medición de procesamiento visual.

H2. Los arquitectos tienen un rendimiento cognitivo superior al común de la población en test de medición de capacidad conceptual y semántica.

2. ¿̇Existe correlación positiva entre percepción visual, imaginería mental, procesamiento semántico y presencia de creatividad en las soluciones de diseño arquitectónico?

H1. Los resultados apreciados como creativos en diseño son el resultado de una lata tasa de interacción entre percepción visual, imaginería mental y activación semántica.

3. ¿̇Desde el punto de vista cognitivo, a que está vinculada la creatividad en la práctica del diseño arquitectónico, al procesamiento visual o procesamiento semántico?

$\mathrm{H} 1$. Los resultados apreciados como creativos son el resultado de una alta tasa de procesamiento visual por parte de los arquitectos.

\section{DISEÑO EXPERIMENTAL Y MÉTODOS DE ANÁLISIS}

A partir de una variación a la técnica de doble disociación para estudios en cognición humana, se definió que un grupo de arquitectos resolvería dos tipos de tareas a fin de obtener dos conjuntos distintos de datos que pudiesen ser revisados por dos grupos diferentes de evaluadores, asegurando la independencia entre las evaluaciones y el análisis de datos. El primer conjunto de tareas, alineado con los tres procesos mentales en estudio, fue una batería de tres conocidos test que miden percepción visual, imaginería mental y categorización semántica, a saber: el test stroop, el test de rotación mental, y el test de categorización semánticos, lo que junto a una encuesta básica en la que se consultó por años de experiencia, y preferencia de medios de trabajo (croquis, computador, maquetas) generó el primer conjunto de datos del estudio.

La segunda tarea fue desarrollar un proyecto de diseño arquitectónico. El proyecto de diseño consistió en un «museo para la historia de la tecnología» ubicado en el campus principal del Instituto Tecnológico de Georgia. Situar el proyecto en el centro del campus aseguró la condición de que todos los participantes conocieran el terreno. El programa arquitectónico entregado consistió de:

- Una plaza pública $\left(380 \mathrm{~m}^{2}\right)$

- Áreas verdes $\left(300 \mathrm{~m}^{2}\right)$

- Hall de acceso $\left(200 \mathrm{~m}^{2}\right)$

- Puesto de venta de entradas $\left(30 \mathrm{~m}^{2}\right.$

- Tres salas de exposición $\left(500 \mathrm{~m}^{2} \mathrm{c} / \mathrm{u}\right.$.)

- Un teatro $\left(500 \mathrm{~m}^{2}\right)$

- Una cafetería y tienda de regalos $\left(50 \mathrm{~m}^{2}\right)$

- Servicios sanitarios $\left(40 \mathrm{~m}^{2}\right)$

- Oficina y sala de reuniones $\left(50 \mathrm{~m}^{2}\right)$

- Bodega $\left(500 \mathrm{~m}^{2}\right)$

- Total: $3.290 \mathrm{~m}^{2}$

Los participantes tuvieron 20 minutos para presentar sus propuestas de diseño y croquis y dibujos a mano alzada fueron el único medio de presentación aceptado. Se facilitaron todos los materiales; lápices, croqueras, un set de 6 fotos 


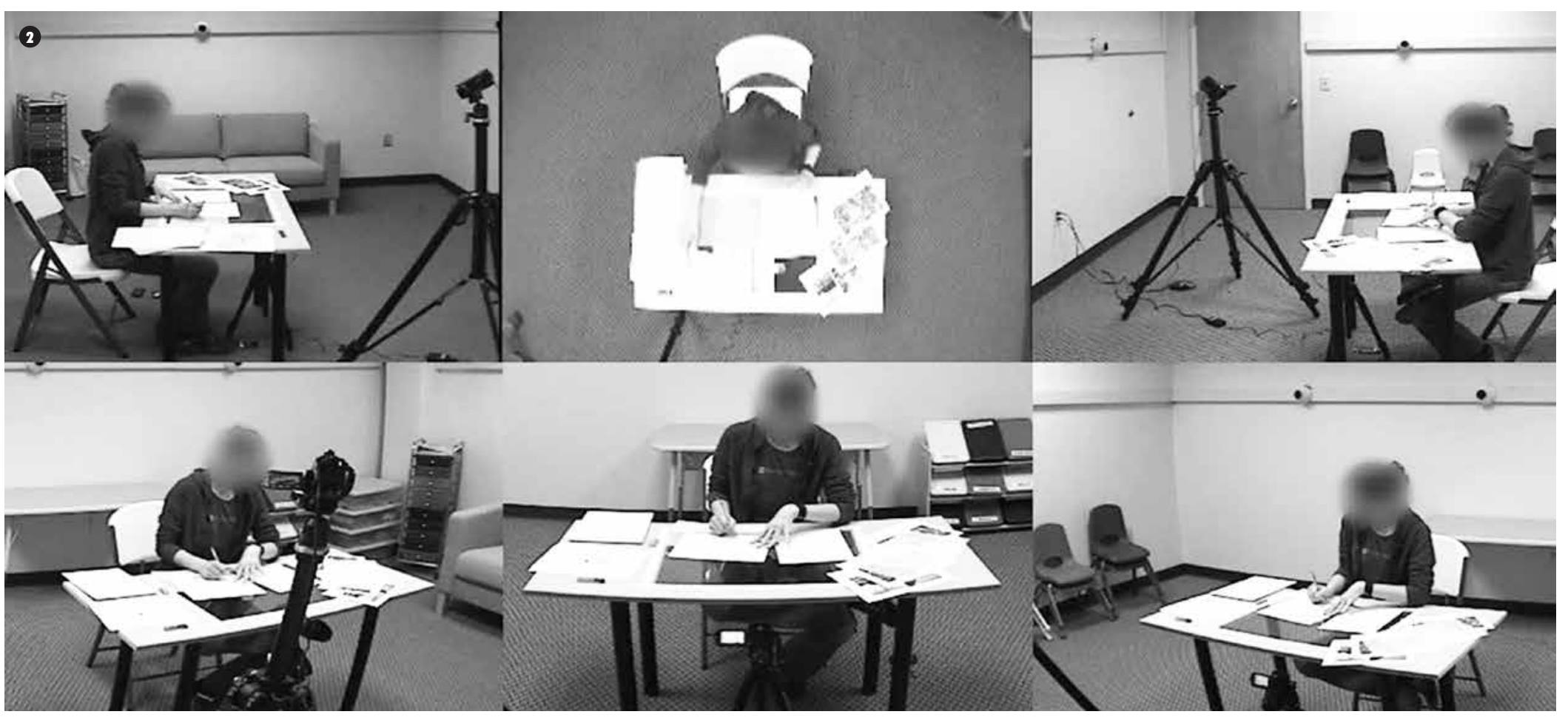

del sitio y una planta de emplazamiento escala $1: 100$. Los croquis hechos por los arquitectos constituyeron el segundo grupo de datos.

Adicionalmente, durante esta fase, se les pidió a los arquitectos que pensaran en voz alta mientras desarrollaron el proyecto de diseño, proceso que fue grabado en audio/video para realizar posteriormente un análisis de protocolos de diseño (Figura 2). Se predispuso que el análisis de protocolos se corroboraría con dos estudiantes de doctorado en cognición del diseño, con experiencia en este tipo de estudios, mientras que los proyectos de diseño serían evaluados por un jurado doble conformado por 3 profesores de arquitectura y 3 profesores de psicología. Los miembros del jurado trabajaron en forma anónima y sin contacto entre ellos para no influir en sus criterios de evaluación a los proyectos de diseño, usando la técnica de medición de creatividad desarrollada por Teresa Amabile, una de las dos técnicas más conocidas y aceptadas actualmente a nivel mundial por la psicología y las ciencias cognitivas. Mientras que el test de Torrance se centra en cuatro aspectos básicos, a saber fluencia, flexibilidad, originalidad y elaboración (Torrance, 1962), el método desarrollado por Amabile propone una medición a través de escala Likert para 22 dimensiones de apreciación de creatividad, lo que permite un análisis granulométrico más fino del fenómeno creativo (Amabile, 1996).

\section{RESULTADOS}

\section{TEST COGNITIVOS}

Contrariamente a lo predicho en las hipótesis, el rendimiento de los arquitectos estuvo completamente adentro de los márgenes considerados comunes para la población estándar. Ninguno de los 16 voluntarios que participaron en el estudio sobresalió en rendimiento cognitivo en términos de percepción visual, habilidades de imaginería mental, ni conceptualización semántica. Las FigurAs 3A, 4A Y 5 A muestran ejemplos de los test y las Figuras $3 \mathrm{~B}, 4 \mathrm{~B}$ Y $5 \mathrm{~B}$ muestran los resultados promediados que obtuvieron los participantes en dichos test.

\section{ANÁLISIS DE PROTOCOLOS}

A fin de analizar los procesos de diseño de los arquitectos, el audio y video capturado fue transcrito y dividido en segmentos de acuerdo a los quiebres y cambios en el foco de atención demostrado por sus acciones y habla (Suwa \& Tversky, 1997). Esta técnica, conocida como análisis de protocolos, es la más comúnmente usada en este tipo de estudios (HinChai, K. \& Xiao, X., 2011 ) desde que Ericsson y Simón la desarrollaran en la década de los cuarenta (Ericsson \& Simon, 1947). La TABLA 1 muestra un ejemplo de una sección de una transcripción ya segmentada.

Posteriormente, dos estudiantes de doctorado asignaron, en forma independiente, los segmentos de las transcripciones a las distintas categorías en estudio: percepción visual, imaginería visual y activación de conocimiento semántico. Los segmentos en que los sujetos mencionaban específicamente observaciones provenientes de los esquemas y croquis que estaban realizando fueron categorizados como 
3. A: Ejemplo del test Stroop en columna derecha. Los participantes deben indicar el color del texto y no lo que está escrito.

B: Resultados del test Stroop. Valores promediados para los 16 arquitectos dentro de rangos normales para el común de la población.

4. A: Ejemplo del test de rotación mental. Los participantes deben indicar si la figura del lado derecho es la misma figura que aparece en el lado izquierdo -rotada espacialmente- o es una figura distinta.

B: Resultados del test rotación mental. Valores promediados para los 16 arquitectos absolutamente dentro de los rangos normales para el común de la población. Lo normal es demorarse más en determinar si las figuras son distintas que si son iguales.

5. A: Ejemplo del test de prototipos o categorización semántica. Primero se muestra el prototipo a los participantes, quienes deben decidir si las imágenes posteriores corresponden o no al prototipo mostrado inicialmente.

B: Resultados del test de categorización semántica. Valores promediados para los 16 arquitectos absolutamente dentro de los rangos normales para el común de la población. Los arquitectos no fueron ni más rápidos ni más lentos y detectaron correctamente el $70 \%$ de las variaciones del prototipo, al igual que el común de la población.

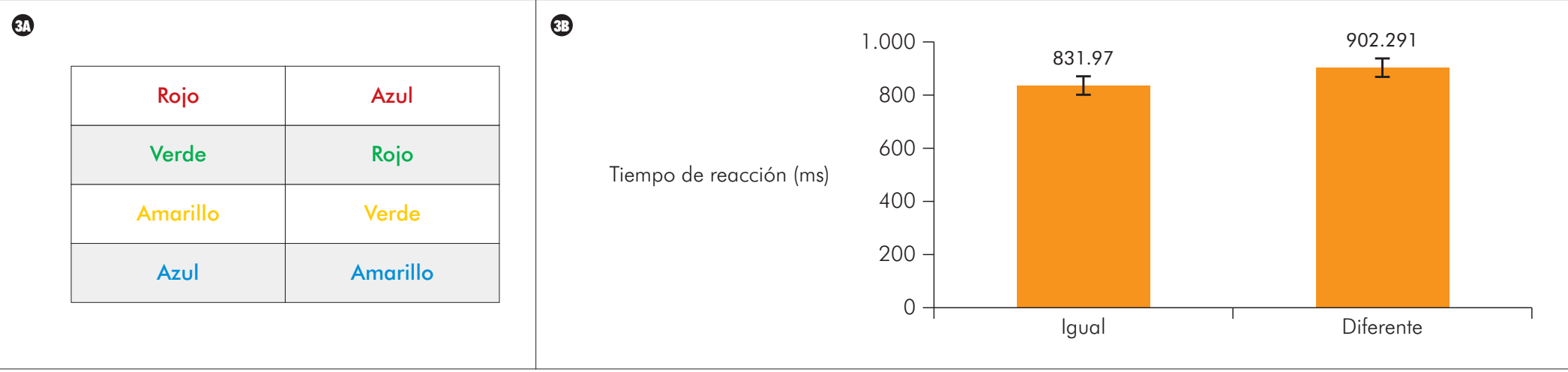

(1)

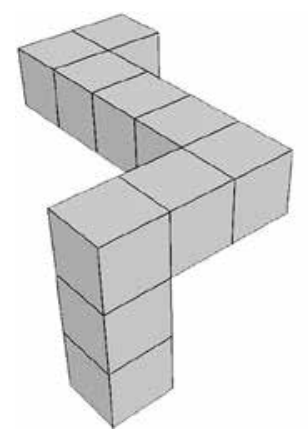

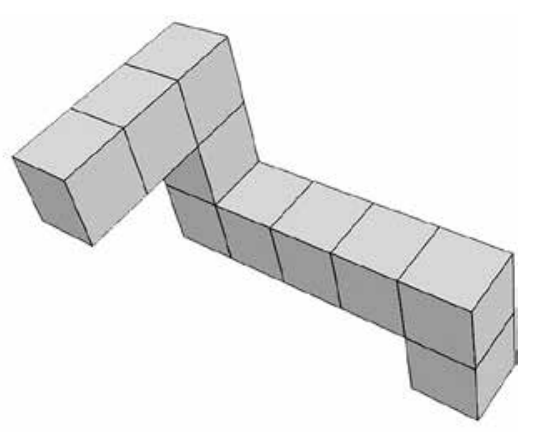

(18)

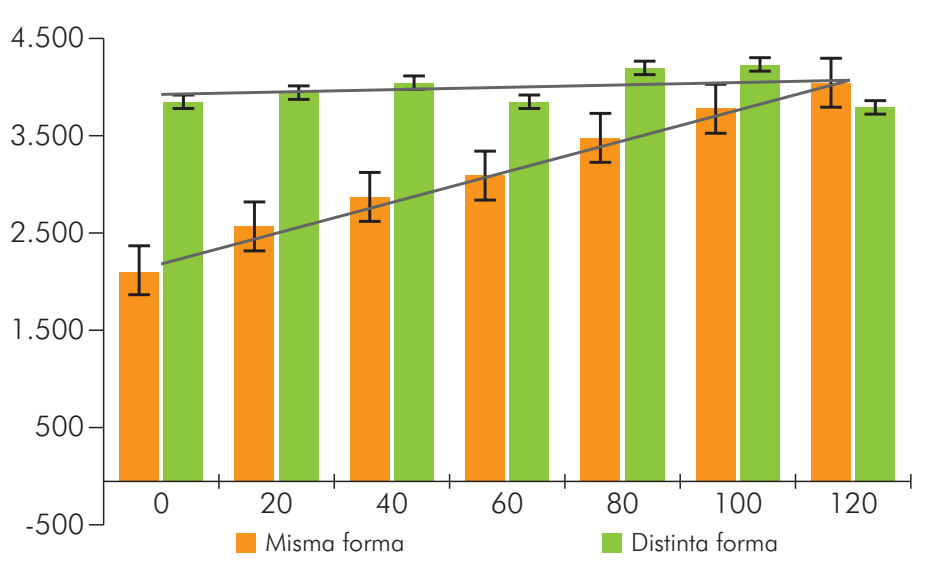

(3)
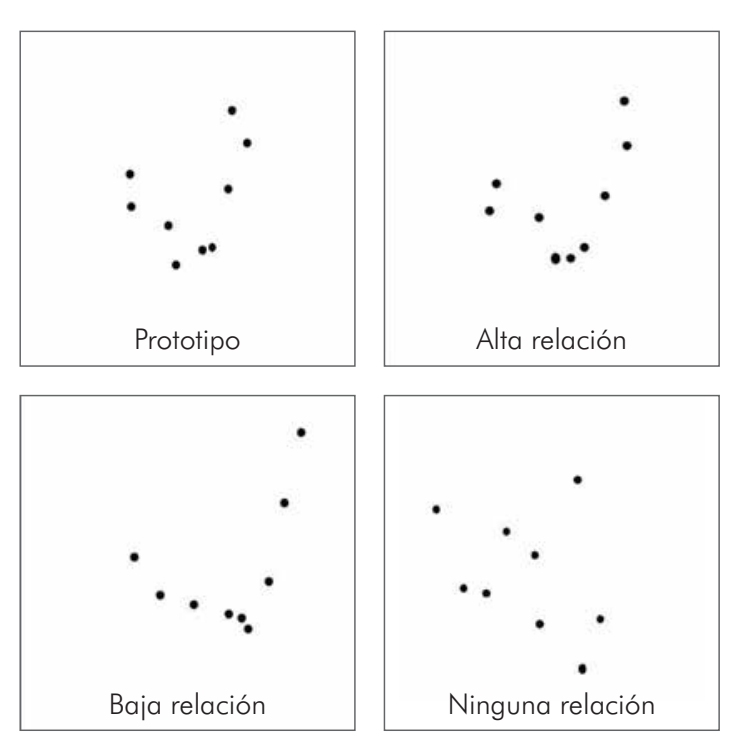

(B)

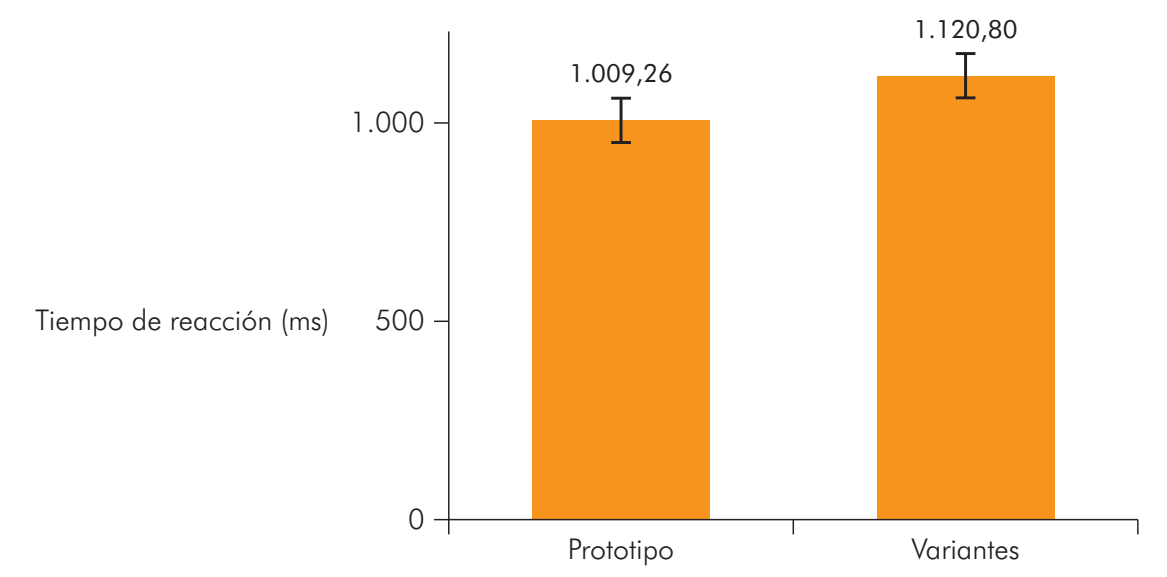


6. Cantidad de episodios en las distintas categorías de procesamiento cognitivo detectados por individuo.

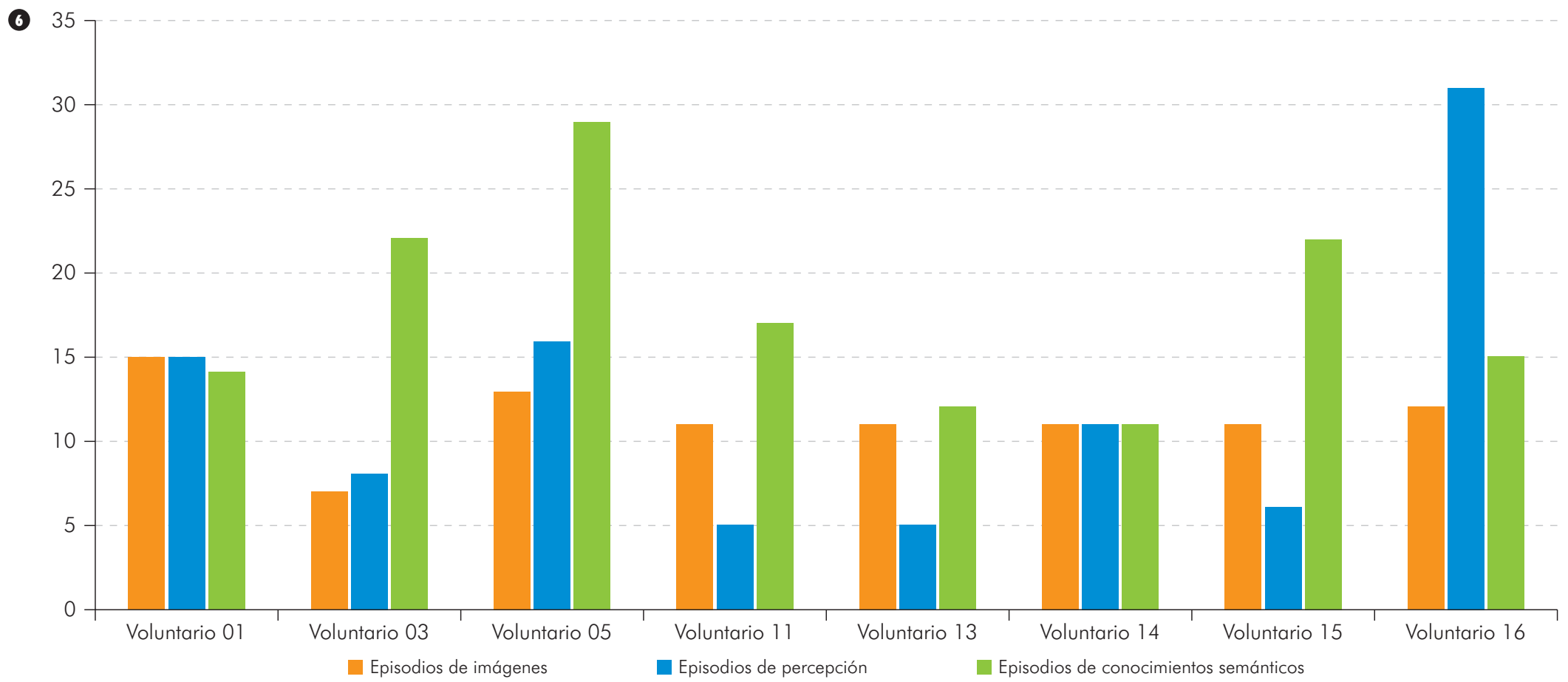

episodios de percepción visual, por ejemplo: «No sé por qué hice una figura cuadrada... podría haber comenzado con un círculo, pero hice un cuadrado...». De la misma manera, los segmentos en que los arquitectos describían imágenes mentales en relación al proyecto fueron catalogados como episodios de imaginería mental, por ejemplo: «la cubierta va a ser como un manto verde, como una especie de colina verde...». Finalmente los segmentos en que específicamente mencionaban información que recordaban o sabían fueron categorizadas como episodios de activación de representaciones en su red semántica, por ejemplo: «los baños van a estar ubicados aquí sirviendo el hall principal...». Ante la enorme cantidad de datos generados por el diseño del experimento, se optó por seleccionar 8 de los 16 casos en base dos criterios, un mínimo de cinco años de experiencia de práctica profesional, y la presencia de un mínimo de 5 episodios en cada categoría cognitiva. La FIGURA 6 muestra los resultados de la cantidad de episodios de percepción visual, imaginería mental y activación de la red de conocimiento semántico para los 8 arquitectos seleccionados.

TABLA 1

Ejemplo de transcripción del audio segmentado según cambios en el foco atencional del individuo

\begin{tabular}{ccl}
6 & $1: 19.7-1: 31.8$ & $\begin{array}{l}\text { you are surrounded by little hills here and the buildings are all higher than } \\
\text { this point... }\end{array}$ \\
\hline 7 & $1: 31.7-1: 42.4$ & $\begin{array}{l}\text { so, maybe is like ahhh...like a monument or something vertical... like an icon } \\
\text { you could have in the middle of campus }\end{array}$ \\
\hline 8 & $1: 42.3-1: 45.2$ & $\begin{array}{l}\text { so, maybe this thing is like that... rotated... and the ground is here... and the } \\
\text { time line is going up... so... }\end{array}$ \\
\hline 9 & $1: 45.1-2: 17.6$ & $\begin{array}{l}\text { thinking another alternative could be... something like wires... because wires } \\
\text { are the main component of technology for me... }\end{array}$ \\
\hline
\end{tabular}

Nota: El estudio se realizó en el idioma inglés por ser el idioma materno de los participantes.

\section{EVALUACIÓN DE NIVEL DE CREATIVIDAD PRESENTE EN LOS PROYECTOS}

Seis profesores, 3 arquitectos y 3 psicólogos evaluaron los proyectos desarrollados por los 8 arquitectos seleccionados, siguiendo la rúbrica de evaluación para creatividad de Teresa Amabile. Dicha rúbrica descompone el fenómeno creativo en 22 dimensiones medibles, cada una de ellas, de acuerdo a una escala tipo Lickert (Amabile, 1996). De estas, dos dimensiones, creatividad y novedad en la idea, se seleccionaron como componentes principales de creatividad. "Creatividad» fue evaluada como el grado en el cual los proyectos resultaban creativos a los ojos del evaluador, mientras que «Novedad en la idea» correspondió al grado en que los proyectos mostraban una idea original. De esta forma se buscó evaluar en forma independiente 
7. Promedios en escala de 1 a 5 de las evaluaciones asignadas por psicólogos y arquitectos a las propuestas de diseño en la segunda etapa de análisis.

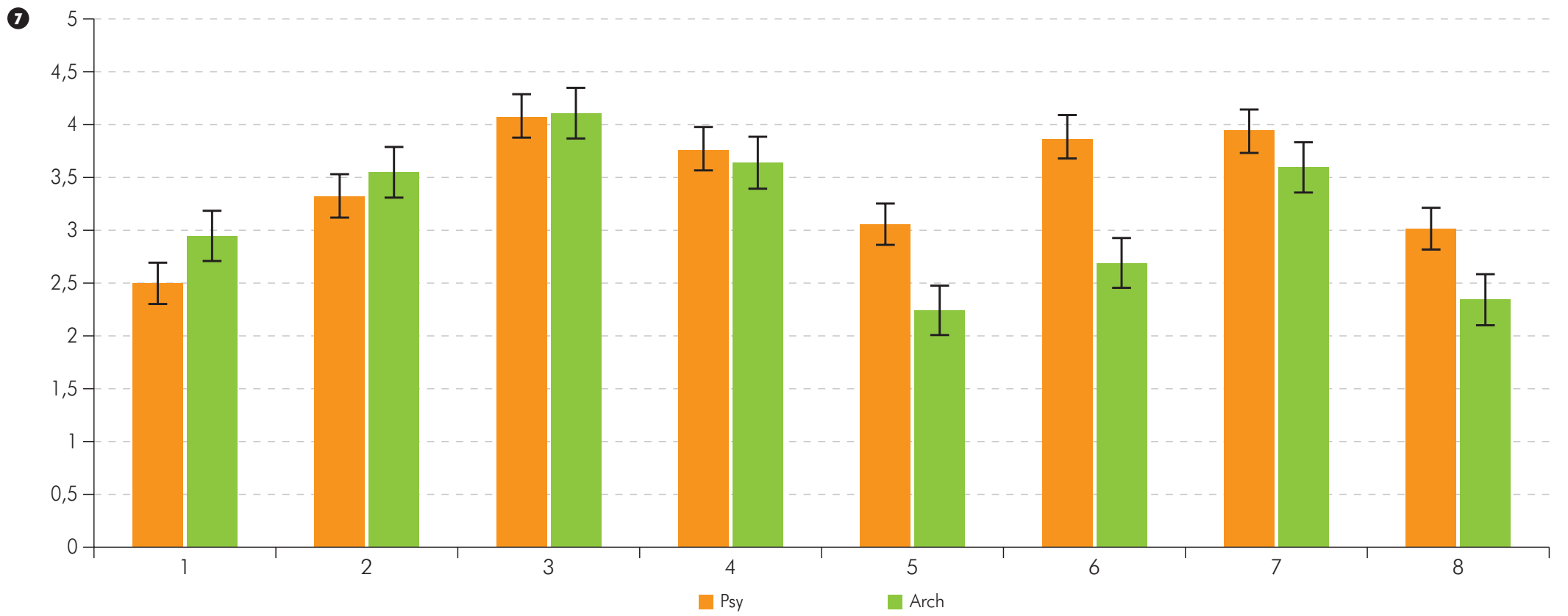

por un lado si los proyectos eran creativos en sí mismos y por otro si la idea de diseño propuesta era novedosa. Sorprendentemente, los resultados del proceso de evaluación de los proyectos arrojó una alta correlación entre lo que psicólogos y arquitectos consideran soluciones creativas en arquitectura, con solo dos casos que escaparon a dicha observación. Como corresponde a este tipo de estudios se calculó el coeficiente de confiabilidad interevaluador (Coeficiente $\alpha$ ), lo que arrojó valores de «1.0» para creatividad y «0.66» para novedad en la idea a los ojos de los arquitectos, mientras que los psicólogos promediaron «1.0» para ambas dimensiones. El coeficiente $\alpha$ general total fue «0.83» para creatividad y «1.0» para novedad. Los promedios normalizados de las evaluaciones asignadas por psicólogos y arquitectos se muestran en la Figura 7 y la confiabilidad de las evaluaciones en la TABLA 2.

Finalmente, es importante hacer notar que ante una definición de trabajo propuesta para «creatividad»-el grado en el cual el proyecto en su totalidad es creativo a los ojos del evaluador- el hecho de que los arquitectos asignaran valores más bajos en creatividad a los

TABLA 2

Coeficiente $\alpha$ para «Creatividad» y «ldea Novedosa» en los dos grupos de evaluadores

\begin{tabular}{lcccc} 
& Arch. Creativity & Arch. Novel Idea & Psych. Creativity & Psych. Novel Idea \\
\hline Arch. Creativity & 1.0000 & 0.8536 & 0.5447 & 0.7335 \\
\hline Arch. Novel Idea & 0.8536 & 1.0000 & 0.6834 & 0.8127 \\
\hline Psych. Creativity & 0.5447 & 0.6834 & 1.0000 & 0.8916 \\
\hline Psych. Novel Idea & 0.7335 & 0.8127 & 0.8916 & 1.0000
\end{tabular}

proyectos que los psicólogos, sugiere primero que arquitectos y psicólogos no entienden lo mismo por proyectos creativos, lo que puede ser explicado por diferencia de expertiz en sus respectivos campos de estudio, y segundo que la condición de expertiz -en el caso de los evaluadores arquitectos- podría gatillar mayor exigencia frente a dicha noción de creatividad.

\section{RESULTADOS GENERALES}

Finalmente se realizó un análisis multivarianza (MANOVA) considerando los resultados de las dimensiones de creatividad antes seleccionadas, el número de episodios que arrojó el análisis de los protocolos y los años de experiencia de los participantes. El resultado muestra una clara correlación entre la presencia de creatividad a los ojos de los evaluadores y el número de episodios catalogados como activación de la red de contenidos semánticos de los arquitectos con un valor $P$ de $p<0,0074$. Ante este inesperado resultado, se corrió un segundo test estadístico en el que la variable años de experiencia fue excluida expresamente para descartar la presunción de que arquitectos con más años de experiencia podrían ser evaluados como más creativos. Dicho test mostró que la correlación entre los dos componentes principales de la creatividad y el número de episodios de activación semántica aún era significante con 
$r=0,82$ y $p=0,021$, lo que permitió descartar la presunción anterior. Los resultados del test de multivarianza se muestran en la TABLA 3.

\section{DISCUSIÓN Y CONCLUSIONES}

Los resultados de esta investigación indican que la educación formal en arquitectura no mejora habilidades cognitivas -que se podrían pensar son claves para el proyectar- como la percepción visual, imaginería mental, o el procesamiento semántico-conceptual. En el presente estudio, arquitectos expertos con una media de 12 años de práctica profesional tuvieron un desempeño cognitivo equivalente al de personas, sin entrenamiento en las disciplinas del diseño, en los tres test cognitivos administrados.

Tampoco se encontraron correlaciones significativas entre las capacidades visuales, ni imaginativas ni perceptuales, en términos de producir proyectos más creativos, mientras que una mayor activación de representaciones semánticas alojadas en la memoria de largo plazo de los arquitectos se correlacionó fuertemente con soluciones de diseño evaluadas como más creativas. El análisis detallado de las representaciones de conocimiento semántico presentes en dicho episodios indica que los arquitectos se aproximan a los problemas de diseño usando principalmente dos estrategias para formular sus propuestas: comenzar desde el sitio o emplazamiento, o comenzar desde el programa.

En el presente estudio, 8 de los dieciséis voluntarios comenzaron el ejercicio analizando el programa arquitectónico, y 7 usaron como punto de partida el lugar. Solo 1 arquitecto usó una idea externa, ajena al encargo de diseño para formular su propuesta: un concepto de diseño. Esto sugiere que los arquitectos descansan primariamente en dos estrategias para hacer la primera formulación de diseño. Por una parte manejan un repertorio de posibilidades de agrupamiento y organización programático que les permite tomar las primeras decisiones clave para organizar

TABLA 3

Resultados del test de multivarianza (MANOVA)

\begin{tabular}{lcccc} 
& P.C. Creativity & Imagery Ep. & Perceptual Ep. & Sem. Knowledge Ep. \\
\hline P.C. Creativity & & -0.0450 & -0.4460 & 0.8657 \\
\hline Imagery Ep. & -0.0450 & & 0.3192 & -0.0016 \\
\hline Perceptual Ep. & -0.4460 & 0.3192 & & 0.3312 \\
\hline Semantic Ep. & 0.8657 & -0.0016 & 0.3312 &
\end{tabular}

propuestas de diseño arquitectónico. En este caso el ordenamiento general de la distribución programática es el objetivo a alcanzar, y las relaciones topológicas y la conectividad de espacios emergen como el problema a resolver. Así, los arquitectos buscan establecer y fijar ubicaciones, proporciones y distancias entre los elementos componentes del programa arquitectónico. La segunda estrategia da cuenta de la formulación del problema proyectual usando el terreno como punto de partida del proyecto. Cuatro temáticas surgieron como patrones en el desarrollo de las propuestas de los arquitectos que siguieron esta línea de trabajo: las condiciones topográficas, la relación entre los patrones de circulación y los bordes del sitio, las características naturales del terreno en cuanto a ventilación y asoleamiento, y las vistas desde y hacia el terreno. Lamentablemente no se detectaron patrones conducentes a establecer una jerarquía en términos de elegir algunas de estas 4 variables sobre las otras al momento de formular propuestas de diseño. Tampoco se detectaron otro tipo de tópicos usados por los arquitectos cuando enfrentaban el subproblema «sitio» durante la formulación de sus propuestas de diseño, lo que hace pensar que la interacción de estos cuatro componentes conforma la idea central, o núcleo, de lo que los arquitectos entienden por terreno desde el punto de vista de las representaciones mentales consolidadas en su memoria de largo plazo.

La interacción y presencia constante del conjunto de representaciones vinculadas a las ideas del programa y del sitio en los episodios de uso de conocimiento semántico, indica que estos son componentes críticos del stock de conocimiento que aprenden, desarrollan y usan los arquitectos para la formulación de sus propuestas de diseño, mientras que el «referente conceptual o concepto» solo juega el rol de detonador de representaciones semánticas aledañas en la red de contenidos alojada en la memoria de largo plazo de los arquitectos.

Respecto del procesamiento visual y acorde con la literatura especializada en cognición visual (Finke, 1989; Kosslyn 1994; Farah, 2000), es interesante mencionar que los participantes ocuparon mayor tiempo y cantidad de episodios analizando propiedades perceptuales visuales tales como ubicación, tamaños y relaciones entre elementos geométricos representando los espacios solicitados por el programa, que propiedades espaciales propiamente tales, como podrían haber sido las proporciones internas de los espacios que estaban siendo diseñados: su altura, escala o profundidad. Esto nos recuerda nuevamente la pregunta sobre la naturaleza del espacio según lo entienden los arquitectos, planteada en los antecedentes previos expuestos al comienzo de este artículo. Especulando una posible respuesta a esta pregunta, tal vez dicha naturaleza sea semántica desde el punto de vista cognitivo. Por supuesto, esto queda por estudiarse.

\section{AGRADECIMIENTOS}

Agradezco al programa Mecesup II del Ministerio de Educación de la República de Chile, a la Facultad de Arquitectura y Urbanismo de la Universidad de Chile y al programa Fullbright del Gobierno de los 
Estados unidos de Norteamérica por financiar parcial e indirectamente este estudio dentro del contexto de los estudios doctorales que persigue en el Instituto Tecnológico de Georgia, Georgia Tech. Asimismo agradezco al Digital Building Lab y su director, profesor Charles «Chuck» Eastman, así como a los académicos responsables del Spatial Innovation Group, investigadores Jonathan Shaw y Matthew Swarts quienes me han dado la oportunidad de trabajar como ayudante de investigación permitiéndome cofinanciar mis estudios doctorales. De la misma manera agradezco al Dr. Mario Romero, Posdoctoral Fellow en el Health System Institute por facilitarme el acceso al Laboratorio de Comportamiento Humano de dicho instituto donde realice la captura de datos de este estudio. Mención aparte merecen los 16 arquitectos, 2 estudiantes de doctorado y 6 profesores en los departamentos de Arquitectura y Psicología del Instituto Tecnológico de Georgia que colaboraron gratuitamente con horas de trabajo sometiéndose a test psicológicos, diseñando proyectos, colaborando con el análisis de datos y completando las evaluaciones de los proyectos. A todos ellos, que no puedo mencionar explícitamente de acuerdo al protocolo aprobado de investigación, mis más sinceros agradecimientos. Todos ustedes saben quiénes son. Finalmente no puedo dejar de agradecer a los profesores Paul Verhaeghen, Nancy Nersessian y Sonit Bafna por su incansable colaboración y supervisión de mi trabajo, así como por los innumerables comentarios y correcciones que los tres hicieron a los múltiples borradores previos a mi «Qualifying paper», trabajo que da origen a este artículo resumido para la revista de arquitectura. Ellos hicieron mi trabajo mejor. Las falencias y errores remanentes son solo responsabilidad mía.

\section{BIBLIOGRAFÍA}

Akin, O. (1982). Representation and Architecture. In O. Akin and E. F. Weinel. Eds. Representations and Architecture. Information Dynamics Inc.

Amabile, T. (1996). Creativity in context: Update to the social psychology of creativity. West View Press.

Chi, M. (1997). Quantifying Qualitative Analysis of Verbal Data - A Practical Guide. The Journal of the Learning Sciences, Vol. 6, N³, pp. 271-315.

Collins, A., and Loftus, E. F. (1975). A SpreadingActivation theory of semantic processing. Psychological Review Vol. 82, pp. 407-428.

Cross, N., and Dorst, K. (1997). Creativity in Design: Analyzing and Modeling the Creative Leap. Leonardo, Vol. 30. No 4. pp. 311-317.

Dorst, K., and Cross, N. (2001). Creativity in the design process: co-evolution of problem-solution. Design Studies, Vol. 22, № 5, 425-437.

Eastman, C. (2001). New directions in design cognition: Studies of representation and recall. In C. Eastman, W. Newstetter, M. McCracken (Eds.) Design knowing and learning: Cognition in design education, Elsevier.

Ericsson, K. A., and Simon, H. A. (1947). Protocol Analysis: Verbal Reports as Data, Ed. Bradfort.

Ericsson, K. A., and Charness, N. (1994). Expert performance: Its structure and acquisition. American Psychologist, Vol. 49, N 8, pp. 725 747.

Ericsson, K. A., Krampe, R., and Tesch-Roemer, C. (1993). The Role of Deliberated Practice in the Acquisition of Expert Performance. Psychological Review, Vol. 100, №3, pp. 363-406.

Farah, M. (1994). Does Visual Attention Select Objects or Locations? Journal of Experimental Psychology. Vol.123. $N^{\circ}$ 2. pp. 146-160.

Farah, M. (2000). The neural bases of mental imagery. In M. S. Gazzaniga (Ed.), The new cognitive neurosciences. MIT Press. Cambridge, Mass.

Finke, R. A. (1989). Principles of Mental Imagery. MIT Press. Cambridge, Mass.

Gazzaniga, M., Ivry, R., and Mangun, G., (1998). Cognitive neuroscience: The biology of the mind (Third Edition). W.W. Norton \& Company, New York NY.
Hin-Chai, K., and Xiao, X. (2012). Understanding design research: A bibliometric analysis of Design Studies (1996-2010). Design Studies. Vol. 33, I. 1 , pp. 24-43.

Kosslyn, S. (1994). Image and Brain. MIT Press. Cambridge Mass.

Kozbelt, A. (2001). Artists as experts in visual cognition. Visual Cognition. Vol. 8, № 6, pp. 705-723.

Lawson, B. (2001). Language of Space. Architectural Press.

Lawson, B. (2004). How Designers Think, Fourth Edition: The Design Process Demystified. Architectural Press.

Lawson, B., and Dorst, K. (2009). Design Expertise. Architectural Press.

Pignocchi, A. (2010). How the intentions of the draftsman shape perception of a drawing. Consciousness and Cognition. Vol. 19, N ${ }^{\circ} 4$, 887-898.

Quillian, M. R. (1966). Semantic Memory. Unpublished doctoral dissertation. Carnegie Institute of Technology. Reprinted in part in Minsky, M., Ed. Semantic information processing. Cambridge, Mass. MIT Press.

Roseman M., and Gero, J. (1993). Creativity in design using a design prototype approach. In J. Gero and M. L. Maher (Eds.) Modeling Creativity and Knowledge-Based Creative Design. Ed. Hillsdale.

Seeley, W., and Kozbelt, A. (2008). Art, artists, and perception: a model for pre-motor contributions to perceptual analysis and form recognition. Philosophical Psychology. Vol. 21, I. 2, pp. 149 171.

Suwa, M., and Tversky, B., (1997). What do architects and students perceive in their design sketches? A protocol analysis. Design Studies. Vol. 18, I. 4, pp. 385-403.

Torrence, E. P., (1962). Guiding Creative Talent. Prentice-Hall

Tulving, E. (1972). Episodic and semantic memory. In Tulving, E. and Donaldson, W. (Eds.), Organization of Memory. Academic Press.

Warrington, E. (1978). Two Categorical Stages of Object Recognition. Perception, Vol 7. № 6. pp 695-705. 